\title{
A NEW METHOD FOR PRIORITIES OF THE HIERARCHIC SYSTEM WITH INNER DEPENDENCE
}

\author{
WangHuei, GuanJiayu, WuYiping \\ Tianjin Institute of Textile Science and Technology
}

ABSTRACT

In this paper, a new method for calculating the priorities of Hierarchic system with inner dependence based on the principle of hierarchical composition is proposed. It's different from Saaty's supermatrix method and is an improvement of the ratio scale approach.

\section{$\rightarrow$ INTRODUCTION}

The priorities of general system with feedback can be calculated by means of supermatrix (4). This method can also be used in calculating the priorities of the hierarchic system with inner dependence (HSID), but it needs much calculation and lacks level's intuitiveness. In fact, There is still the highest level in HSID, even though the elements in each level are dependent upon themselves, So that the priorities of HSID are in essence the same as that of the hierarchic system with independence (HSI). In this paper, a new method for calculating the priorities of HSID based on the principle of hierarchical composition is proposed. It improves the ratio scale approach (3). Through this method. We can get the same conclusion as the one calculated by supermatrix. This method is very clear, simple and intuitive, and the number of calculations can also be cut down greatly. This method can calculate the priorities of the hierarchic system both with inner dependence and independence.

In this paper, the principles of the method are first given in Section 2 , the method will be described in detail in Section 3. In Section 4, we will prove the effectiveness of this method. And finally, an applied example will be given as an illustration for this method.

\section{二. PRINCIPLES OF THE METHOD}

Consider a HSID which has been decomposed into n ciusters or components $C(1), C(2), \ldots C(n)$, $\mathrm{N}^{-}$is the total number of the system s elements, $\mathrm{n}(\mathrm{k})$ is the number of the elements in the kth level, $1(k)=n(1)+n(2)+\cdots+n(k), E(c(k))=\{l(k-1)+1, l(k-1)+2, \cdots \cdots, l(k)\}$ are the elements set in the kth level (where we use the element's subscript to represent the element itself). The $s-$ step priority for a pair of elements $i, j$ is given by

$$
w(i, j)(s)=\sum_{m=1}^{N} w(i, m) \cdot w(m, j)^{(s-1)}
$$

where $w(i, m)$ is the impact priority of the ith element to the mth element in the system. $w(m, j)^{(0-1)}$ is the $s-1$ step priority of the mth element to the jth element in the system

Theorem 1: In HSID, the s-step impact priorities of any pair of elements in the same level are 
oniy relative to that of the elements in this level, not relative to that of elements in other level. i.e.

$$
w(i, j)^{(s)}=\sum_{m=1(k-1)+1}^{l(k)} w(i, m) \cdot w(m, j)^{(m-3)}, \quad V i, j \in E(c(k))
$$

Proof, In terms of $(2-1)$, we derive

$$
\begin{aligned}
w(i, j)(s) & =\sum_{m=1}^{N *} w(i, m) \cdot w(m, j)(s-1) \\
& =\sum_{m=1}^{(k-1)} w(i, m) \cdot w(m, j)^{(2-1)}+\sum_{m=1(k-1)+1}^{1(k)} w(i, m) \cdot w(m, j)^{(s-1)}+\sum_{m=1(k)+1}^{l(m)} w(i, m) \cdot w(m, j)^{(m-1)}
\end{aligned}
$$

When $m>l(k-1), m \in E(C(k+1)) \cup E(C(k+2)) \cup E(C(k+3)) \cup \ldots E(C(n))$. We know $i \in E(C(k))$. so the impact priority of the ith element on the mth element is equal to zero, i.e., $w(i, m)=0$, the third item of $(2-3)$ is equal to zero. When $m<1(k-1), m \in E(C(k-1)) \cup E(C(k-2)) \ldots \cup E(C$ (1)). We know $j \in E(C(k))$, so the impact priority of the mth element to the jth element is equal to zero, i.e., $\mathbb{}(\mathrm{m}, \mathrm{j})=0$, the first item of $(2-3)$ is also zero, so $(2-3)$ becomes:

$$
w(i, j)(s)=\sum_{m=1(k-1)+1}^{(4)} w(i, m) \cdot w(m, j)(n-1)
$$

\section{Theorem 1 holds}

Theorem 2, In HSID, the s--step impact priorities of each element in $\mathrm{C}(\mathrm{k})$ to any element in $\mathrm{C}$ $(k-1)$ are onjy relative to that of elements in these two levels, not relative to that of elements in other level, i.e.

$$
w(i, j)^{(s)}=\sum_{m=1(k-2)+1}^{\mid(k)} w(i, m) \cdot w(m, j)^{(s-l)}, \quad V i \in E(C(k)), V j \in E(C(k-1))
$$

Proof: According to (2-1), we get

$$
\begin{aligned}
& w(i, j)^{(1)}=\sum_{m=1}^{N^{*}} w(i, m) \cdot w(m, j)^{(--1)}
\end{aligned}
$$

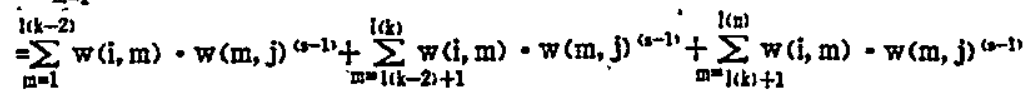

Simulating the proof of Theorem 1. we can prove the first and the third items of $\{2-5\}$ are equal to zero, so $(2-5)$ becomes:

$$
w(i, j)(s)=\sum_{m=l(k-2)+1}^{l(k)} w(i, m) \cdot w(m, j)^{(m-1)}
$$

So, Theorem 2 holds.

\section{三. THE METHOD FOR SETTING UP THE LIMITING IMPACT PRIORITIES}

1. The limiting impact priorities of HSID with two levels

Now we konw the s-step impact priorties of each element in $C(k)$ to any element in $C(k-1)$ satisfy $(2-4)$. we use supermatrix to represent $(2-4)$ as

$$
W(k)^{(s+1)}=W(k) \cdot W(k)^{(s)}
$$


where

$$
C(k-1) \quad C(k)
$$

8

$$
\left.W(k)=\begin{array}{ll}
C(k-1) \\
W(k-1 . k-1) & 0 \\
W(k \cdot k-1) & W(k \cdot k)
\end{array}\right)
$$

$W(k-1, k-1), W(k, k)$ are respectively impact priority matrix of $C(k-1)$ and $C(k), W(k, k-1)$ is impact priority matrir of each element in c(k) to any element in $\mathrm{C}(\mathrm{k}-1)$ (see $: 4 ;$ ).

We assign a different weight value to each of the levels, according to the importance of their contribution to the system, thns we get the weighting supermatrix.

$$
\bar{W}(k)=\left\{\begin{array}{cc}
a(k-1, k-1) W(k-1, k-1) & 0 \\
a(k, k-1) W(k, k-1) & W(k, k)
\end{array}\right\}
$$

8

then (3-1) becomes

$$
\bar{W}(k)^{(0+1)}=\overline{\mathrm{W}}(k) \cdot \overline{\mathrm{W}}(\mathrm{k})^{(s)}
$$

According to Theorem 2, we know that the s-step impact priorities of $\mathrm{C}(\mathrm{k})$ to $\mathrm{C}(\mathrm{k}-1)$ are only reiative to that of the elements in these two levels, so $C(k)$ and $C(k-1)$ can be regarded as a HSID with two levels. In terms of Saaty's priority theory of system with feedback (4). through calculating the fimiting value of $(3-4)$, we can get the limiting inpact priorities of $C(k)$ to $C(k-1)$ $\left(W(k, k-1)^{-}\right)$. In case of $W(k, k-1)^{*}$. the HSID consisting of $C(k)$ and $C(k-1)$ can be simplified as HSI consisting of $C(k)$ and $C(k-1)$. For examplez

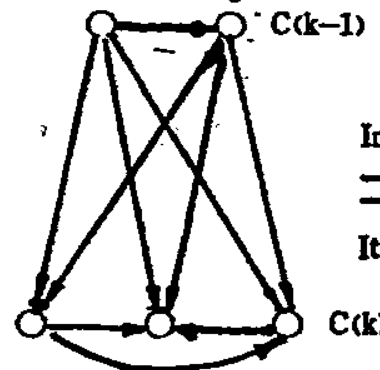

(In case of $W(k, k-1))$
In case of $W(k, k-1)^{*}$

It's can be simpified

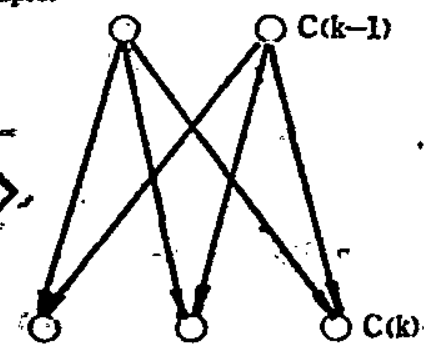

c. (In case of $\left.\mathbf{W}(\mathrm{k}, \mathrm{k}-1)^{\prime 2}\right)$

2. The fimiting priorities of HSID with n levels

Suppose we have a HSID, shown in Figure I, by means of above method, we calculate the liniting impact priorities of $C(n)$ to $C(n-1)\left(W(n, n-1)^{\cdots}\right)$. In case of $W(n, n-1) \%$. the system can be simpified, shown in Figure 2 With the simplified system, we continue to calculate the limiting impact priorities of $C(n-1)^{2}$ to $C(n-2)\left(W(n, n-1)^{-1}\right)$ and can simplified the system further. Identical with that of above, cakcuate $W(n-2, n-3)^{*}, \ldots \ldots . W(2,1)^{* *}$ in sequence. In the end, the system call be simplified as a HSI, shown in Figure 3, thus, the limiting impact priorities of the 
alternative level to the goal level can be calculated as follows:

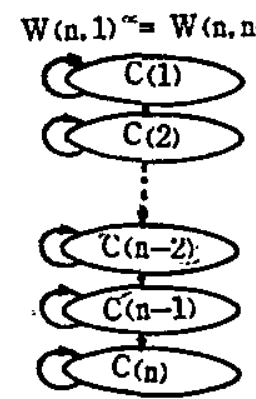

Figure 1

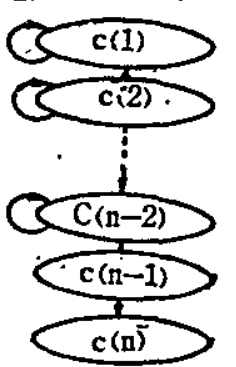

Figure 2

四. THE PROOF OF THE METHOD'S EFFECTIVENESS

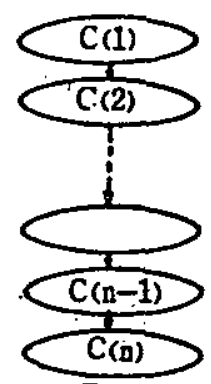

Figure 3

The effectiveness of this method can be proved when $W(n, n)$ is a normal matrix.

Lemma 1, For HSID with two levels, when $W(n, n)$ is a normal matrix, note: $W(n, n)^{\text {ce }}=$ lim $W$ $(n, n)^{2}$, then, the limiting impact priorities of the alternative level to the goal level exist and can be expressed as follows:

$$
W(n, 1)^{\alpha}=W(n, n)^{\infty} \cdot a(n, 1) \cdot W(n, 1)(1-a(1,1) W(1,1))^{-1}, \quad(n=2)
$$

Proof is given in paper $\{1\}$

Theorem 3t For HSID with $n$ levels, When $W(n, n)$ is a normal matrix, note $W(n, n)^{*}=\lim$ $W(n, n)$, then the limiting impact priorities of the alternative level to the goal level exist and can be expressed as follows.

$$
\begin{aligned}
W(n, 1)^{x} & =W(n, n)^{x} \cdot a(n, n-1) \cdot W(n, n-1)(1-a(n-1, n-1) W(n-1, n-1))^{-1} \cdots \ldots a a(2,1) \\
& \cdot W(2,1)(1-a(1,1) W(1,1)\}^{-t}
\end{aligned}
$$

Proof: The system is shown in Figure 1. For $C(n)$ and $C(n-1)$, we can establish their weighting supermatrix as follows

$$
\bar{W}(n)=\left(\begin{array}{lc}
a(n-1, n-1) W(n-1, n-1) & 0 \\
a(n, n-1) W(n, n-1) & W(n, n)
\end{array}\right)
$$

Because $C(n)$ and $C(n-1)$ can be regarded as a HSID with two levels, and $W(n, n)$ is a normal matrix, so according to Lemma 1 , we know

$$
\mathrm{W}(\mathrm{n}, \mathrm{n}-1)^{<}=\mathrm{W}(\mathrm{n}, \mathrm{n})^{-<} \cdot \mathrm{a}(\mathrm{n}, \mathrm{n}-1) \mathrm{W}(\mathrm{n}, \mathrm{n}-1)(\mathrm{I}-\mathrm{a}(\mathrm{n}-1, \mathrm{n}-1) \mathrm{W}(\mathrm{n}-1, \mathrm{n}-1))^{-1} .
$$

In case of $W(n, n-1)^{*}$, the system can be simplified, shown in figure 2. For $C(n-1)$ and $C(n-2)$, establishing their weighting supermatrix. the elements of $C(n-1)$ are now independent upon themselves, so $W(n-1, n-1)=I$, thus 


$$
\bar{W}(n-1)=\left[\begin{array}{lc}
a(n-2, n-2) W(n-2, n-2) & 0 \\
a(n-1, n-2) W(n-1, n-2) & 1
\end{array}\right]
$$

According to Lemma 1 , we get

$W(n-1, n-2)^{\wedge}=a(n-1, n-2) \cdot W(n-1, n-2) \div(1-a(n-2, n-2) W(n-2, n-2)\}^{-i}$

Just the same as above. we get

$W(k, k-1)^{\prime \alpha}=a(k, k-1) \cdot W(k, k-1) \cdot(1-a(k-1, k-1) W(k-1, k-1))^{-1}$

$$
\text { * }(k=n-2, n-3, \cdots, 2)
$$

In the end, the system can be simplified as HSI, shown in Figure. 3. Through the synthesizing formulation of $\mathrm{HSl}_{\text {s }}$

We get

$$
W(n, 1)^{\infty}=W(n, n-1)^{\infty} \cdot W(n-1, n-2)^{\infty} \ldots \ldots W(2,1)^{\infty}
$$

$$
\begin{aligned}
W(n, 1)^{\alpha} & =W(n, n)^{\infty} \cdot a(n, n-1) \cdot W(n, n-1)\left(I-a(n-1, n-1) W(n-1, n-1)^{-1} \ldots \ldots a(2,1)\right. \\
& \cdot W(2,1)(1-a(1,1) W(1,1))^{-1}
\end{aligned}
$$

So Theorem 3 holds.

From theorem 3, we know that this new method can lead to the same conclusion as the one calculated by supermatrix. Next, we turn to some special cases:

A: When the elements in each levels are independent upon themselves, i.e., the system is HSI. $W(i, i)=I, a(i, i)=0,(i=1,2, \ldots, n), W(n, n)^{\infty}=I, \quad(4-2)$ can be simplified as follows:

$$
W(n, 1)^{<}=W(n-1, n-2) \cdot W(n-2, n-3) \ldots \ldots W(2,1)
$$

This is just the composited formulation of HSI, so HSI can be regarded as a special case of HSID.

$B_{2}$ When the elements in the alternative level are independent upon themselves, and the goal level has oniy one element, $W(n, n)^{\alpha}=1, a(1,1)=0,(4-2)$ can be simplified as follows:

$$
W(n, 1)^{*}=a(n, n-1) W(n, n-1)(I-a(n-1, n-1) W(n-1, n-1)\}^{-1} \cdots a(2,1) W(2,1) \ldots
$$

C: According to the synthesizing formulation $(4-2)$, the priorities of the alternative level to any criterion level $\mathrm{C}(\mathrm{k})$ can be calculated as follows:

$$
W(n, k) \propto=W(n, n-1)^{\infty} \cdot W(n-1, n-2)^{\infty} \ldots . . \cdot W(k+1, k)^{\star}
$$

and the priorities of any criterion level $\mathrm{C}(\mathrm{k})$ to the goal level can also be calculated as follows

$W(k, 1)^{*}=W(k, k-1)^{*} \cdot W(k-1, k-2)^{*} \ldots \ldots W(2,1)^{*}$

$$
\begin{aligned}
=W(k, k)^{*} \cdot a(k, k-1) \cdot W(k, k-1)(1-a(k-1, k-1) W(k-1, k-1))^{-1} \cdots \ldots \cdot a(2,1) \\
\\
\cdot W(2,1) \cdot\left(I-a(1,1) \cdot W(1,1)^{-1} .\right.
\end{aligned}
$$

D. When $W(n, n)$ is not a normal matrix, this method stij holds, we don't discuss these cases in this paper.

\section{五. APPLIED EXAMPLE}

The Comprehensive Estimation of University's Education levels. The model is shown in Figure 4 


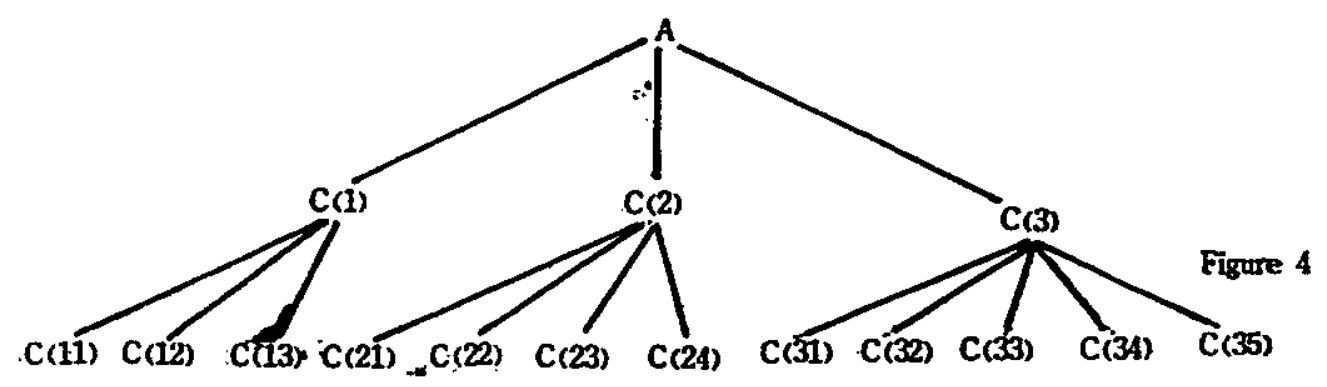

A Comprehensive Estimation of University's Education level

C(1) Accomplishments of Teaching and Scientific Research

C(2) Investment and Benefit

C(3) Management Level

C(11) Undergraduate Students Training

C(12) Graduate Students Training

C(13) Benefit and Effect of Scientific Research

C(21) Teacher's Level and Training

$C$ (22) Investment for Instruments, Equipments, Materials etc.

\section{C(23) Public Welfare}

C(24) Returns on Investment

C(31) School-Boand's Administration

C(32) Moral Education

C(33) Management of Teaching

C(34) Management of Scientific Research

C(35) Management of Auxiliary Facilities

We calculate the limiting impact priorities of alternatives to a goal as follows:

Stepl: For the 3th level and the 2th level, establish their weighting supermatrix as thus

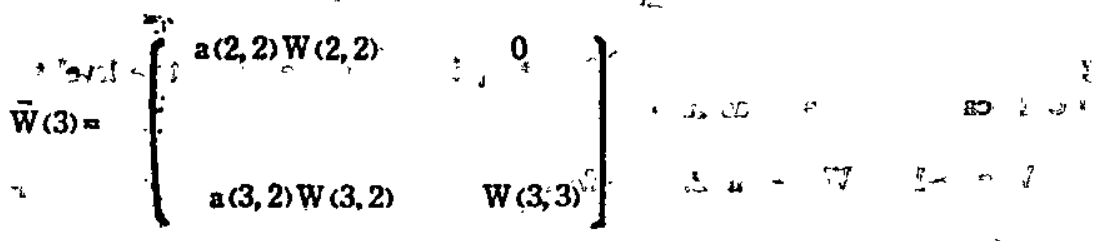

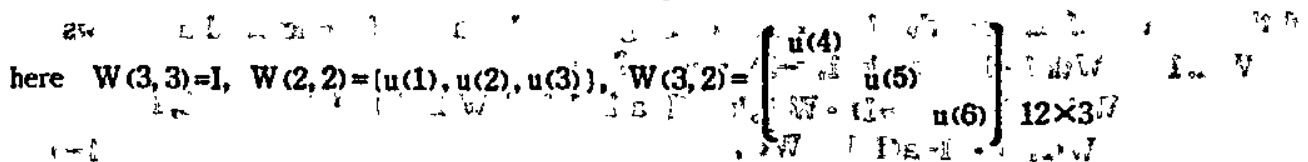

$\mathrm{u}(1)=(0.6608,0.2081,0.1311)^{\top}, \mathrm{u}(2)=(0.1365,0.6350,0.2385)^{\top}, \mathrm{u}(3)=(0,0.25,0.75)^{\top}, \mathrm{u}(4)=$ (0. $5,-0.25,0.25)^{t} u(5)=(0.481,0.243 .0 .166,0.109)^{\tau}, u(6)=\left(0.313,0.313,0.176,0.099^{t}\right.$ $0.099)^{\top}, \mathrm{a}(3,2)=0.8, \mathrm{a}(2,2)=0.2$.

Calculating in $\overline{\mathrm{W}}(3)$, we get;

$W(3,2)^{\approx}=(a(1), a(2), a(3))$

$\mathrm{a}(1)=\left(0.4676,0.2338,0.2338,0.0138,0.0069,0.0041,0.0031,0.0005,0.0005,0.002,0.001^{3} 0.001\right)^{x}$

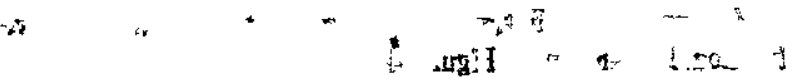

